[white paper]

Diamond Open Access

\title{
Números representáveis
}

\author{
Colaboração Matemática Aberta ${ }^{1}$
}

17 de Dezembro de 2020

\section{Resumo}

Este artigo apresenta a demonstração de um dos teoremas que estão no livro Proofs from THE BOOK [1].

palavras-chave: números representáveis, primos, congruências

A versão mais atualizada deste artigo está disponivel em https://osf.io/cq48x/download

\section{Como ser coautor(a) deste artigo?}

1. Envie-nos suas observações e responda às seguintes perguntas.

2. Quais discussões você adicionaria a este artigo?

3. Você encontrou algum erro ou argumento inconsistente? Dê uma justificativa detalhada.

${ }^{1}$ Todos os autores com suas afiliações aparecem no final deste artigo. 


\section{Introdução}

4. Informações complementares sobre algumas propriedades que apresentamos neste trabalho estão disponíveis em [1-3].

5. Sabe-se que o menor número primo é 2, o único par. Os demais primos são ímpares e podem ser escritos na forma $4 m+1$ ou $4 m+3$, com $m \in\{0,1,2,3, \ldots\}[2,3]$.

6. O objetivo deste artigo é mostrar que um número será representável somente se todos os fatores primos da forma $4 m+3$ na decomposição de $n$ possuírem expoentes pares.

\section{Teorema}

7. Um numero natural $n$ pode ser representado como uma soma de dois quadrados se, e somente se, todo fator primo de $n$ da forma $p=4 m+$ 3 aparece com um expoente par na decomposição de $n$ em números primos. [1]

\section{Preliminares}

8. Sejam $\mathbb{N}_{0}=\{0,1,2,3, \ldots\}$ e $\mathbb{N}=\{1,2,3, \ldots\}$.

9. Para $k \in \mathbb{N}$, diz-se que $a$ e $b$ são congruentes se os restos da divisão euclidiana por $k$ são iguais, e escreve-se: $a \equiv b(\bmod k)$.

10. Pela divisão euclidiana, adaptada de [4], $\forall n \in \mathbb{Z}, \exists m, r \in \mathbb{Z}$, únicos, tal que $n=k m+r, r \in\{0,1,2, \ldots, k-1\}$.

11. Para $k=4, n=4 m+r$, com $r \in\{0,1,2,3\}$.

12. Por (11), $n=4 m+0, n=4 m+1, n=4 m+2$ ou $n=4 m+3$, ou seja, $n \equiv 0,1,2$ ou $3(\bmod 4)$, respectivamente. 
13. Por (12), tem-se que os números da forma:

(i) $4 m+0$ e $4 m+2$ são pares, pois $n=4 m+0=2 \cdot 2 m=2 m^{\prime}$ e $n=4 m+2=2 \cdot(2 m+1)=2 m^{\prime \prime}, \operatorname{com} m^{\prime}, m^{\prime \prime} \in \mathbb{N}_{0} ; \mathrm{e}$

(ii) $n=4 m+1$ e $n=4 m+3$ são ímpares, pois $n=4 m+1=2 \cdot 2 m+1=$ $2 m^{\prime}+1$ e $n=4 m+3=2 \cdot(2 m+1)+1=2 m^{\prime \prime}+1, \operatorname{com} m^{\prime}, m^{\prime \prime} \in \mathbb{N}_{0}$.

14. Por (12) e (13), todo primo $p \neq 2$ é da forma $4 m+1$ ou $4 m+3$.

15. Teorema: Sendo p um número primo, a equação $x^{2}+y^{2}=p$ tem solução se, e somente se, $p=2$ ou $p=4 m+1$.

16. Para demonstração de (15), veja [5].

\section{Prova do teorema (7)}

17. Definição: Um número $n \in \mathbb{N}$ é representável quando pode ser escrito como a soma dos quadrados de dois números naturais de $\mathbb{N}_{0}$.

18. $5=2^{2}+1^{2}, 9=3^{2}+0^{2}, 13=3^{2}+2^{2}$ e $41=4^{2}+5^{2}$ são exemplos de números representáveis.

19. Seja $n \in \mathbb{N}$ um número representável, com $n=x^{2}+y^{2}, x, y \in \mathbb{N}_{0}$.

20. Lema: O número natural $n$ pode ser decomposto da seguinte forma: $n=2^{\alpha} \cdot p_{1}^{\alpha_{1}} \cdot p_{2}^{\alpha_{2}} \cdot \ldots \cdot p_{r}^{\alpha_{r}} \cdot q_{1}^{\beta_{1}} \cdot q_{2}^{\beta_{2}} \cdot \ldots \cdot q_{s}^{\beta_{s}}, \operatorname{com} p_{i}=4 m+1 \equiv 1(\bmod 4)$ e $q_{j}=4 m+3 \equiv 3(\bmod 4), i=1,2, \ldots, r$ e $j=1,2, \ldots, s$, com $m \in \mathbb{N}_{0}$.

21. Por exemplo, em $700=2^{2} \cdot 5^{2} \cdot 7$, tem-se $p=5$ e $q=7$.

22. Note que $n=1=1^{2}+0^{2}$ e $n=2=1^{2}+1^{2}$ são representáveis.

23. Por $(14)$ e $(20)$, se $p$ é primo, $p \neq 2$, então $p \equiv 1(\bmod 4)$ ou $p \equiv$ $3(\bmod 4)$. 
24. Por (9), tem-se
(i) $x=0 \Rightarrow x^{2}=0^{2}=0 \equiv 0(\bmod 4)$;
(ii) $x=1 \Rightarrow x^{2}=1^{2}=1 \equiv 1(\bmod 4)$;
(iii) $x=2 \Rightarrow x^{2}=2^{2}=4 \equiv 0(\bmod 4)$;
(iv) $x=3 \Rightarrow x^{2}=3^{3}=9 \equiv 1(\bmod 4)$.

25. Assim, por (24), $\forall x \in \mathbb{N}_{0}$,
(i) se $x$ é par $\Rightarrow x^{2}=(2 w)^{2}=4 w^{2} \equiv 0(\bmod 4), w \in \mathbb{N}_{0}$;
(ii) se $x$ é ímpar $\Rightarrow x^{2}=(2 w+1)^{2}=4\left(w^{2}+w\right)+1=4 m+1 \equiv 1(\bmod 4)$, $m=w^{2}+w, w \in \mathbb{N}_{0}$.

26. Por (25), $\forall x, y \in \mathbb{N}_{0}$ e $(x, y)=1$,

(i) $x^{2}+y^{2} \equiv 0(\bmod 4)$, se $x$ e $y$ são pares;

(ii) $x^{2}+y^{2} \equiv 1(\bmod 4)$, se $x$ e $y$ possuem paridade distintas;

(iii) $x^{2}+y^{2} \equiv 2(\bmod 4)$, se $x$ e $y$ são ímpares.

27. Por $(15)$ e $(26 . i i), p=x^{2}+y^{2} \equiv 1(\bmod 4)$, ou seja, $p=4 m+1$ é representável.

28. Se $n_{1}=x_{1}^{2}+y_{1}^{2}$ e $n_{2}=x_{2}^{2}+y_{2}^{2}$ são representáveis, então

$$
\begin{aligned}
n_{1} \cdot n_{2} & =\left(x_{1}^{2}+y_{1}^{2}\right) \cdot\left(x_{2}^{2}+y_{2}^{2}\right) \\
& =x_{1}^{2} x_{2}^{2}+x_{1}^{2} y_{2}^{2}+x_{2}^{2} y_{1}^{2}+y_{1}^{2} y_{2}^{2} \\
& =x_{1}^{2} x_{2}^{2}+2 x_{1} x_{2} y_{1} y_{2}+y_{1}^{2} y_{2}^{2}+x_{1}^{2} y_{2}^{2}-2 x_{1} x_{2} y_{1} y_{2}+x_{2}^{2} y_{1}^{2} \\
& =\left(x_{1} x_{2}+y_{1} y_{2}\right)^{2}+\left(x_{1} y_{2}-x_{2} y_{1}\right)^{2}
\end{aligned}
$$

é representável.

29. Se $n=x^{2}+y^{2}$ é representável, então $n \cdot w^{2}=\left(x^{2}+y^{2}\right) \cdot w^{2}=(x w)^{2}+$ $(y w)^{2}, w \in \mathbb{N}$, é representável.

30. Se o primo $q=4 m+3\left|n=x^{2}+y^{2} \Rightarrow q\right| x$ e $q \mid y$. 
31. Por (30), se $q^{2}\left|n=x^{2}+y^{2} \Rightarrow q^{2}\right| x^{2}$ e $q^{2} \mid y^{2}$.

32. O número $q=4 m+3$ não é representável.

(i) Suponha, por contradição, que $q=4 m+3$ seja representável.

(ii) Por $(15), x \neq 0(\bmod q) \Rightarrow \exists \bar{x} ; x \cdot \bar{x} \equiv 1(\bmod q)$.

(iii) Por (30), $q \mid x^{2}+y^{2} \Rightarrow x^{2}+y^{2} \equiv 0(\bmod q)$.

(iv) Multiplicando a congruência $x^{2}+y^{2} \equiv 0(\bmod q)$ por $\bar{x}^{2}$, tem-se:

$$
\begin{aligned}
\left(x^{2}+y^{2}\right) \cdot \bar{x}^{2} \equiv 0 \cdot \bar{x}^{2}(\bmod q) & \Rightarrow(x \bar{x})^{2}+(\bar{x} y)^{2} \equiv 0(\bmod q) \\
& \Rightarrow 1+(\bar{x} y)^{2} \equiv 0(\bmod q),
\end{aligned}
$$

que é impossível para $q=4 m+3$.

33. De (20), tem-se $q_{j}^{\beta_{j}}=(4 m+3)^{\beta_{j}}, j=1,2, \ldots, s$.

34. Por (33),

(i) se $\beta_{j}$ é ímpar: $\beta_{j}=2 \beta_{j}^{\prime}+1 \Rightarrow q_{j}^{\beta_{j}}=q_{j}^{2 \beta_{j}^{\prime}+1}=q_{j}^{2 \beta^{\prime}} \cdot q_{j}=\left(q_{j}^{\beta_{j}^{\prime}}\right)^{2} \cdot q_{j}$.

(ii) se $\beta_{j}$ é par: $\beta_{j}=2 \beta_{j}^{\prime} \Rightarrow q_{j}^{\beta_{j}}=q_{j}^{2 \beta_{j}^{\prime}}=\left(q_{j}^{2}\right)^{\beta_{j}}$.

35. Por (32), a igualdade de $(34 . i), q_{j}^{\beta_{j}}=\left(q_{j}^{\beta_{j}^{\prime}}\right)^{2} \cdot q_{j}$, não é representável.

36. De (34), conclui-se que apenas $q_{j}^{2}$ é representável, pois $q_{j}^{2}=q_{j}^{2}+0^{2}$.

37. Portanto, o número natural $n=x^{2}+y^{2}=2^{\alpha} \cdot p_{1}^{\alpha_{1}} \cdot p_{2}^{\alpha_{2}} \cdot \ldots \cdot p_{r}^{\alpha_{r}} \cdot q_{1}^{\beta_{1}} \cdot q_{2}^{\beta_{2}} \cdot \ldots \cdot q_{s}^{\beta_{s}}$ é representável $\Leftrightarrow \beta_{j}$ for par, $j=1,2, \ldots, s$. 


\section{Considerações Finais}

38. Portanto, foi mostrado

(i) que na decomposição em fatores primos do número natural

$$
n=x^{2}+y^{2}=2^{\alpha} \cdot p_{1}^{\alpha_{1}} \cdot p_{2}^{\alpha_{2}} \cdot \ldots \cdot p_{r}^{\alpha_{r}} \cdot q_{1}^{\beta_{1}} \cdot q_{2}^{\beta_{2}} \cdot \ldots \cdot q_{s}^{\beta_{s}},
$$

tem-se que os fatores primos 2 e $p_{i}=4 m+1, m \in \mathbb{N}$, são representáveis;

(ii) que o quadrado de um fator primo e o produto de dois números representáveis também são representáveis;

(iii) que o fator primo $q_{j}=4 m+3, m \in \mathbb{N}_{0}$, é representável somente se possuir um expoente par; e

(iv) por fim, que o número natural $n=x^{2}+y^{2}$ é representável se, e somente se, em sua decomposição, todos os fatores primos da forma $4 m+3$, possuírem expoentes pares.

\section{Ciência Aberta}

O arquivo latex para este artigo, juntamente com outros arquivos suplementares, estão disponíveis [6].

\section{Referências}

[1] AIGNER, Martin; ZIEGLER, Günter M.. Proofs from THE BOOK. 6. ed. Berlim: Springer, 2018. 326 p.

[2] FREIRE, Benedito Tadeu V. Números primos: Os argumentos de euclides e aplicações. CD da Revista do Professor de Matemática (RPM), São Paulo: IME USP, 2012. Disponível em <http://rpm.org.br/cdrpm/11/2.htm>. Acesso em: 26 out. 2020. 
[3] PINTO, Ana Carolina Neves et al. Inteiros que são soma de dois quadrados. 2011. 18 f. Monografia (Especialização) - Curso de Matemática, Departamento de Matemática, Estatística e Computação Científica, Universidade Estadual de Campinas, Campinas - Sp, 2011. Disponível em: <https://cutt.ly/IgQbwsR>. Acesso em: 26 out. 2020 .

[4] HEFEZ, Abramo. Aritmética: coleção profmat. 2. ed. Rio de Janeiro: Sbm, 2016. 288 p.

[5] SANTOS, João Evangelista Cabral dos. Números Inteiros como soma de Quadrados. 2013. 60 f. Dissertação (Mestrado) - Curso de Mestrado Profissional em Matemática em Rede Nacional - Profmat, Departamento de Matemática, Universidade Federal da Paraíba, João Pessoa - Pb, 2013. Disponível em: <https://cutt.ly/agQvc9y>. Acesso em: 26 out. 2020.

[6] Lobo, Matheus P. "Open Journal of Mathematics and Physics (OJMP)." OSF, 21 Apr. 2020.

https://doi.org/10.17605/osf.io/6hzyp

\section{Colaboração Matemática Aberta}

Giordane Lima Santana (autor principal, giordanesantana@hotmail. com $)^{1,2}$ https://orcid.org/0000-0002-2935-3761

Matheus Pereira Lobo (mplobo@uft.edu.br) $)^{1,3}$ https://orcid.org/0000-0003-4554-1372

Davi Santana de Oliveira Silva ${ }^{1,4}$

Hans Müller Silva Oliveira ${ }^{1,5}$

Francisco Djalma da Silva Paulo1,6 
${ }^{1}$ Universidade Federal do Tocantins (Brasil)

${ }^{2}$ Escola Paroquial Luiz Augusto (Tocantins, Brasil)

${ }^{3}$ Universidade Aberta (UAb, Portugal)

${ }^{4}$ Escola Municipal Turma da Mônica (Tocantins, Brasil)

${ }^{5}$ Colégio Invictos (Tocantins, Brasil)

${ }^{6}$ Colégio Militar do Tocantins, Unidade 3 (Tocantins, Brasil) 